\title{
Network Creation Games with Disconnected Equilibria
}

\author{
Ulrik Brandes ${ }^{1}$, Martin Hoefer ${ }^{2, \star}$, and Bobo Nick ${ }^{1}$ \\ ${ }^{1}$ Department of Computer \& Information Science, University of Konstanz \\ \{ulrik.brandes, bobo.nick\}@uni-konstanz.de \\ ${ }^{2}$ Lehrstuhl Informatik I, RWTH Aachen University \\ mhoefer@cs.rwth-aachen.de
}

\begin{abstract}
In this paper we extend a popular non-cooperative network creation game (NCG) [11] to allow for disconnected equilibrium networks. There are $n$ players, each is a vertex in a graph, and a strategy is a subset of players to build edges to. For each edge a player must pay a $\operatorname{cost} \alpha$, and the individual cost for a player represents a trade-off between edge costs and shortest path lengths to all other players. We extend the model to a penalized game (PCG), for which we reduce the penalty for a pair of disconnected players to a finite value $\beta$. We prove that the PCG is not a potential game, but pure Nash equilibria always exist, and pure strong equilibria exist in many cases. We provide tight conditions under which disconnected (strong) Nash equilibria can evolve. Components of these equilibria must be (strong) Nash equilibria of a smaller NCG. But in contrast to the NCG, for the vast majority of parameter values no tree is a stable component. Finally, we show that the price of anarchy is $\Theta(n)$, several orders of magnitude larger than in the NCG. Perhaps surprisingly, the price of anarchy for strong equilibria increases only to at most 4 .
\end{abstract}

\section{Introduction}

The study of distributed network creation with selfish agents has attracted much research interest from various disciplines. A general framework for such an approach was proposed by Jackson and Wolinsky [14]. In their games there are $n$ players and each player is a vertex in a graph. A strategy consists of choosing which incident edges to build. Depending on the network structure there is a payoff for each player, and players adjust their strategy to maximize their payoff. A general finding was that there are games, in which no efficient network is stable for a concept of pairwise stability, which requires bilateral consent to construct a connection. The extensions and adjustments to this model are numerous [13]. In particular, several works extended the model to unilateral link creation and the Nash equilibrium as stability concept [5,9]. A particularly interesting variant was proposed by Fabrikant et al. [11]. In their network creation game (NCG) the

\footnotetext{
* Supported by DFG-Graduiertenkolleg 1298 "AlgoSyn” at RWTH Aachen University.
} 


\begin{tabular}{l|l|l|l|l|l|l|l}
\hline & 1 & $<\frac{4}{3}$ & $<4$ & $<6$ & $\Theta(1)$ & $o\left(n^{\epsilon}\right)$ & $<1+\frac{6 n \log n}{\alpha}$ \\
\hline
\end{tabular}

Fig. 1. Price of anarchy in the NCG

cost of creating an edge is fixed to a parameter $\alpha$. Edge creation is unilateral, and the cost for a player is a trade-off between edge costs and structural network position measured by shortest path distances to all other players. In $[2,8,11]$ the inefficiency of Nash equilibria was quantified using the price of anarchy [15], the ratio of the cost of the worst Nash equilibrium over the cost of a social optimum state. The presently known results on the price of anarchy are summarized in Fig. 1. Other equilibrium concepts were also studied, e.g. pairwise stable equilibria [7], or strong equilibria [3], as well as extensions to more general edge costs or different player cost trade-offs $[1,10,17]$. In network analysis [6], the inverse of the sum of shortest path lengths is one of the most commonly used measures of centrality known as closeness [12]. A problem with closeness is that global connectivity is required for the scores to be comparable. This means that in the NCG for moderate to high edge costs the trade-off is distorted by the enforcement of connectivity. Thus, it was not surprising that trees proved to be a prominent equilibrium structure [11].

In this paper, we remedy this problem by replacing the infinite cost of not being connected by a finite penalty $\beta$. This corresponds directly to a variant of closeness centrality proposed by Botafogo et al. [18], and it was suggested as an open problem in [11]. For special values of $\beta$ it is closely related to a measure called radiality [19]. Our penalized network creation game (PCG) is introduced in Sect. 2. Since the cost of connected equilibria is the same as in the NCG, we are interested in existence, structure, and cost of disconnected Nash equilibria. If $\beta$ is large, Nash equilibria of the PCG are similar to those of the NCG, in particular, they are connected. For smaller $\beta$, disconnected Nash equilibria evolve, and an interesting insight gained from Sect. 3 is that the prevalent tree structures of the NCG are absent whenever $\beta>2$ or $\alpha>1$ (see Theorem 3). In addition, we consider the price of anarchy in Sect. 4 . There are parameter values, for which disconnected Nash equilibria appear but the social optimum is connected, which could lead to an unbounded price of anarchy. However, we show that the price of anarchy in the PCG is always bounded by $O(n)$. In addition, Theorem 4 reveals cases with a tight matching lower bound of $\Omega(n)$. This bound is strictly larger than any bounds for the NCG. In Sect. 5 we consider players that can play joint coordinated deviations and strong equilibria. Unless $\alpha$ and $\beta$ are within a small range, the social optimum is also a strong equilibrium (Theorem 5 ). In Theorem 6 we prove that the price of anarchy for strong equilibria is at most 4. This reveals that in the PCG regular Nash equilibria can be several orders of magnitude more costly than strong equilibria, a question which is still unsolved for the NCG. Due to spacial constraints proofs are sketched or omitted and will be given in the full version of the paper. 


\section{The Model and Initial Results}

The network connection game (NCG) is a tuple $(V, \alpha)$ and can be described as follows. The set of players $V$ is the set of vertices of a graph. Possible edges $\{i, j\} \in V \times V$ have cost $\alpha$. A strategy $s_{i}$ of a player $i$ is a subset $s_{i} \subset V \backslash\{v\}$ and indicates, which edges player $i$ chooses to build. In this way a strategy vector $s$ induces a set of edges between the players. Given a strategy vector $s$ the individual cost for a player $i$ is $c_{i}(s)=\alpha\left|s_{i}\right|+\sum_{j \neq i} \operatorname{dist}_{s}(i, j)$, where $\alpha>0$ and $\operatorname{dist}_{s}(i, j)$ is the length of a shortest-path in the undirected graph $G_{s}=\left(V, E_{s}\right)$ induced by the strategy vector $s$. Note that $G_{s}$ is assumed to be undirected, i.e. each edge can be traversed in any direction, independent of which player pays for it. In the regular connection game $\operatorname{dist}_{s}(i, j)=\infty$ if players $i$ and $j$ are in different components of $G_{s}$. In the penalized network creation game (PCG) we are given a penalty value $\beta>1$, and $\operatorname{dist}_{s}(i, j)=\beta$ for players $i$ and $j$ in different components. A pure Nash equilibrium ( $N E$ ) is a state $s$, in which no player can unilaterally decrease her $\operatorname{cost} c_{i}$ by changing her strategy $s_{i}$. We will restrict our attention to pure equilibria throughout. The social cost $c(s)$ of a state $s$ is simply $c(s)=\sum_{i \in V} c_{i}(s)$. The social optimum state $s^{*}$ is a state with minimum social cost. Note that for the cost of a state it does not matter, which of the two players connected by an edge chose to pay for it, and hence we will sometimes use the graph $G_{s}$ for $s$. States that play an important role in the analysis of the PCG are the empty state $s_{\emptyset}=(\emptyset, \ldots, \emptyset), s_{K}$ corresponding to the complete graph, in which each edge $\{i, j\}$ with $i \neq j$ is paid by player $\min \{i, j\}$, and $s_{Z}$ corresponding to a center-sponsored star, in which one player purchases edges to all other players. Fabrikant et al. [11] show that there is always a pure NE in the NCG and mention that it might be found by iterative improvement steps. Finding a best-response for a player in a NCG, however, was shown NP-hard [11], and this translates to the PCG for sufficiently large penalty cost. In addition, we show that better-response dynamics may cycle, hence the game is no potential game [16]. As the dynamics involve no disconnectivities, the result follows directly for the PCG. Nevertheless, in the PCG there is always a pure NE. This serves as a first insight to motivate the further study of the properties of pure NE in

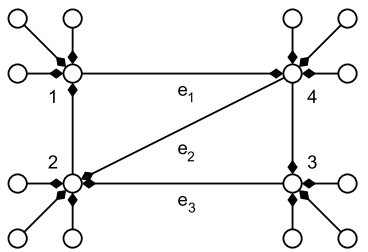

Fig. 2. NCG with $k=4$ and $4<$ $\alpha<6$ with cycling better response iteration. Black dots indicate the player who pays for the edge. the PCG.

Theorem 1. Every PCG has a pure Nash equilibrium, but neither NCG nor $P C G$ are potential games.

Proof. We first disprove the existence of a potential function. For any $\alpha>3$ choose an integer $k$ with $k<\alpha<\frac{3 k}{2}$. Now construct a strategy combination for $n=4 k$ players as depicted in Fig. 2 . The following steps each represent a strict improvement for the players: (1) player 4 removes edge $e_{1},(2)$ player 2 removes 
edge $e_{2},(3)$ player 4 builds edges $e_{1}$ and $e_{2}$. This leads into an isomorphic state, and allows to construct an infinite improvement path. For the proof of existence it can be shown that the following states are NE: for $\alpha \geq \beta-1$ the empty network $s_{\emptyset}$, for $1 \leq \alpha<\beta-1$ the center-sponsored star $s_{Z}$, in which all edges are bought by the center node, and for $\alpha<1$ and $\alpha<\beta-1$ the state $s_{K}$.

\section{Disconnected Equilibria}

In this section we consider existence and structural properties of disconnected NE in the PCG. First, we clarify the existence of disconnected equilibria.

Theorem 2. For $\alpha \geq \beta-1$ the empty graph is always a disconnected NE. For $0<\alpha<\beta-1$ there is no disconnected NE.

Proof. The first part follows from Theorem 1. For the second part consider a player $v$ in a disconnected NE $s$. Let $n_{v}$ be the size of the component of the graph $G_{s}$, in which $v$ is located. Now suppose $v$ changes her strategy by connecting to all $n-n_{v}$ players in other components. Then the change is $\alpha\left(n-n_{v}\right)+(n-$ $\left.n_{v}\right)-\beta\left(n-n_{v}\right)=\left(n-n_{v}\right)(\alpha-(\beta-1))<0$. Hence, under these conditions every player in a disconnected state can decrease her individual cost.

The theorem provides a tight characterization using the empty graph. An interesting issue, however, is to explore whether non-empty disconnected NE are possible, because in many cases the empty graph represents a rather unrealistic prediction for a stable network. Note that a component of $k$ players in a disconnected NE of a PCG with given $\alpha$ and $\beta$ must be a NE in the corresponding NCG with $\alpha$ and $k$ players. A prominent structure that has been identified as NE in the NCG are trees.

Trees. Tree graphs are a structure whose appearance is wide-spread in the NCG $[2,11]$. The following analysis shows that this property is only due to the requirement that a NE must be connected. The following discussion reveals that in the PCG these structures can appear only in very special cases.

Lemma 1. For $\beta>2$ every non-singleton player $v$ in a disconnected NE has at least one incident edge that was created by a different player $w \neq v$.

Proof. Consider a player $v$ in a component $C$ with $k$ players, who pays for all her $d_{v}$ incident edges. As we have a NE, it is not profitable for $v$ to disconnect from $C$, i.e. $\alpha d_{v}+\sum_{w \in C} \operatorname{dist}(v, w) \leq \beta(k-1)$. Consider a different player $v^{\prime} \notin C$ that chooses to connect to all neighbors of $v$. This must not be profitable, so $\alpha d_{v}+\sum_{w \in C} \operatorname{dist}(v, w)+2 \geq \beta k$. Adding the inequalities yields $\beta \leq 2$.

Lemma 2. Suppose there is a disconnected $N E$ with a component $C$ of $k>1$ vertices. If $\alpha>(k-1)(\beta-2)+1$, then for every player $v$ there is an incident edge paid by a different player $w \neq v$. 
Proof. Suppose there is a player $v$ that pays for all her $d_{v} \geq 1$ incident edges. As $v$ does not want to remove all edges, we have $\alpha d_{v}+\sum_{w \in C} \operatorname{dist}(v, w) \leq \beta(k-1)$, and thus $\alpha \leq \frac{1}{d_{v}}\left(\beta(k-1)-\sum_{w \in C} \operatorname{dist}(v, w)\right)$. Every pair of non-neighbor vertices in $C$ has a distance of at least 2 , so $\sum_{w \in C} \operatorname{dist}(v, w) \geq 2(k-1)-d_{v}$. Substitution yields $\alpha \leq(k-1)(\beta-2)+1$ as desired.

Theorem 3. For $\beta>2$ or $\alpha>1$ no component of a disconnected NE is a tree.

Proof. The first bound is a direct consequence of Lemma 1 and the fact that for a tree $|E|=|V|-1$. Thus, for disconnected NE with tree components $\beta \leq 2$, and the second bound follows with Lemma 2.

Non-empty Equilibria. It can be shown that the appearance of currently known NE topologies from the NCG as components in disconnected NE of the PCG is quite limited. The existence of disconnected NE, however, is guaranteed by the empty network. This raises the question under which conditions on $\alpha$ and $\beta$ non-empty disconnected NE can evolve. We first present a positive result.

Lemma 3. For $3 \leq \alpha \leq 4$ and $\beta \leq(\alpha+11) / 5$ a cycle $C_{5}$ of 5 vertices can be a component of a disconnected $N E$.

In contrast to the restricted interval, for which we can show existence, there is an unbounded region of parameter values, for which the empty network is the only disconnected network - in particular if $\alpha$ or $\beta$ are large compared to $n$.

Lemma 4. In a non-empty disconnected $N E$ let $n_{l}$ be the minimum size and diam $_{l}$ the minimum diameter of any non-singleton component. Then (1) $\alpha<$ $12 n_{l} \log n_{l}$, (2) $\beta \leq 1+2 \cdot \operatorname{diam}_{l}$, (3) $\beta<1+14 \sqrt{n_{l} \log n_{l}}$, and (4) if $n>6$, then $\beta<n / 2$.

Proof. We only prove the first three bounds here. For the first bound consider $\alpha \geq 12 n_{l} \log n_{l}$ and a component with $n_{l}$ players. This component must represent a NE in a NCG with the same $\alpha$ and $n_{l}$ players, and thus according to [2] must be a tree. This contradicts Theorem 3 and the bound follows. Now consider a nonempty disconnected NE $s$ for $\beta>2$, and let $C$ be a non-singleton component. As $C$ is no tree, it must contain at least one cycle. Let $U$ be a smallest of all cycles in $C$, and let $v$ be an arbitrary player that constructed some edge $e$ of $U$. Denote by $s^{\prime}$ the state that evolves if player $v$ removes edge $e$. Note that by this removal no additional pair of players gets disconnected. As $s$ is a NE, we have $\alpha \leq \sum_{w \in C}\left(\operatorname{dist}_{s^{\prime}}(v, w)-\operatorname{dist}_{s}(v, w)\right)$. As we have chosen $U$ to be of minimum size, all shortest distances between vertices of $U$ are given by the paths along the cycle. Thus, there is always a vertex $u$, for which the distances in $s$ and $s^{\prime}$ are the same. This yields $\operatorname{dist}_{s^{\prime}}(v, w) \leq \operatorname{dist}_{s^{\prime}}(v, u)+\operatorname{dist}_{s^{\prime}}(u, w)$ $=\operatorname{dist}_{s}(v, u)+\operatorname{dist}_{s}(u, w)$ for all $w \in C$. With $n_{C}=|C|$ we can conclude $\alpha \leq$ $2 n_{C} \cdot \operatorname{diam}(C)-\sum_{w \in C} \operatorname{dist}_{s}(v, w)$. On the other hand, no vertex outside $C$ must be able to profit from a connection to $v$, hence $\alpha+n_{C}+\sum_{w \in C} \operatorname{dist}(v, w) \geq n_{C} \beta$. The last two inequalities deliver the second bound. We know from [11] that $\operatorname{diam}(C) \leq \sqrt{4 \alpha+1}$. Together with the bounds (1) and (2) shown above this implies the third bound. 
In contrast to these bounds, we have not been able to derive any non-empty disconnected NE for values of $\beta>3$. This led us to formulate the following conjecture. Note that our bounds imply that if the conjecture is false, then there must be non-tree NE in the NCG with a diameter of size $\omega(1)$. This seems quite unlikely, as all non-tree NE found so far have diameter at most 3.

Conjecture 1 (Constant Penalty Conjecture). There is a constant $\beta^{\prime}$ such that for $\beta>\beta^{\prime}$ the only disconnected NE is $s_{\emptyset}$.

\section{Price of Anarchy}

In this section we consider the price of anarchy in the PCG. We first consider the social optima of the game. For $\alpha \leq \min \{2,2 \beta-2\}$ the complete graph $s_{K}$ is the optimum. For $\alpha \leq 2$ and $\alpha \geq 2 \beta-2$ the empty graph $s_{\emptyset}$ is the optimum. $s_{\emptyset}$ remains the optimum for $\alpha \geq 2$ and $\alpha \geq \beta n-2(n-1)$. For the remaining range the $\operatorname{star} s_{Z}$ is the optimum. For $\alpha<\beta-1$ we have seen in Theorem 2 that no disconnected NE exists. In addition, it can be shown that in this case a finite penalty for disconnectivity cannot disrupt any NE of the NCG. Hence, for this parameter range the price of anarchy is identical to the NCG. In general, however, the price of anarchy in the PCG can be strictly larger than for the NCG. Fig. 3 provides an overview of the bounds we obtained. Note that all these bounds are in $O(n)$ for the respective parameter values. We concentrate on the case $\max \{2, \beta-1\}<\alpha<\beta n-2(n-1)$, in which disconnected NE can appear and the star is the social optimum.

Theorem 4. For $2 \beta-2 \leq \alpha \leq n \beta-2(n-1)$ the price of anarchy is bounded by $\Theta\left(\frac{n \beta}{\alpha}\right)$ for $\alpha \geq 12 n \log n$ and $O\left(5^{\sqrt{\log n}} \log n+\frac{n \beta}{\alpha+n}\right)$ for $\alpha<12 n \log n$. For $\beta-1 \leq \alpha \leq 2 \beta-2$ the price of anarchy is $\Theta(\min \{\beta, n\})$.

Proof. For the proof of the first bound consider $\alpha \geq 12 n \log n$. According to Lemma 4 in this case every NE is either connected or $s_{\emptyset}$. For $\alpha \geq 12 n \log n$ all connected NE have a constant price of anarchy [2], while $s_{\emptyset}$ leads to an increase and proves our first bound: $\frac{c\left(s_{\emptyset}\right)}{c\left(s_{Z}\right)}=\frac{\beta n}{\alpha+2(n-1)} \in \Theta\left(\frac{n \beta}{\alpha}\right)$. This bound increases from $\Theta(1)$ to $\Theta(n)$ if $\alpha$ drops from $n \beta-2(n-1)$ to $2 \beta-2$. It also shows that the price of anarchy induced by $s_{\emptyset}$ is never more than $O(n)$ for $s^{*}=s_{Z}$ and $\alpha \geq \beta-1$. Another range, for which $s_{\emptyset}$ is the most expensive $\mathrm{NE}$, is $\beta-1 \leq \alpha \leq 2 \beta-2$ with $\beta \geq 7$. Then any directly connected pair induces a cost of $\alpha+2 \leq 2 \beta$. Any indirectly connected pair in a NE induces a

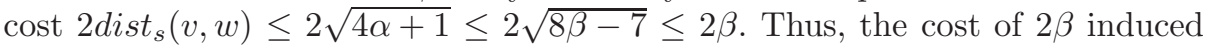
by $s_{\emptyset}$ is maximal for every pair of players. $c\left(s_{\emptyset}\right) / c\left(s_{Z}\right)$ characterizes the price of anarchy and results in $\Theta(\min \{\beta, n\})$, which proves the third bound. For the remaining range with $\alpha<12 n \log n$ there might be worse disconnected $\mathrm{NE}$ than $s_{\emptyset}$. However, components of these NE must be connected NE of smaller NCGs. We bound the price of anarchy for these NE by the fraction for $s_{\emptyset}$ plus the maximum factor of any component NE in the corresponding NCG. With the bound of $5^{\sqrt{\log n}} \log n \in o\left(n^{\epsilon}\right)$ on the price of anarchy for the NCG [8] this proves our second bound $O\left(\max \left\{5^{\sqrt{\log n}} \log n, \min \{n, \beta\}\right\}\right)$, which is at most $O(n)$. 


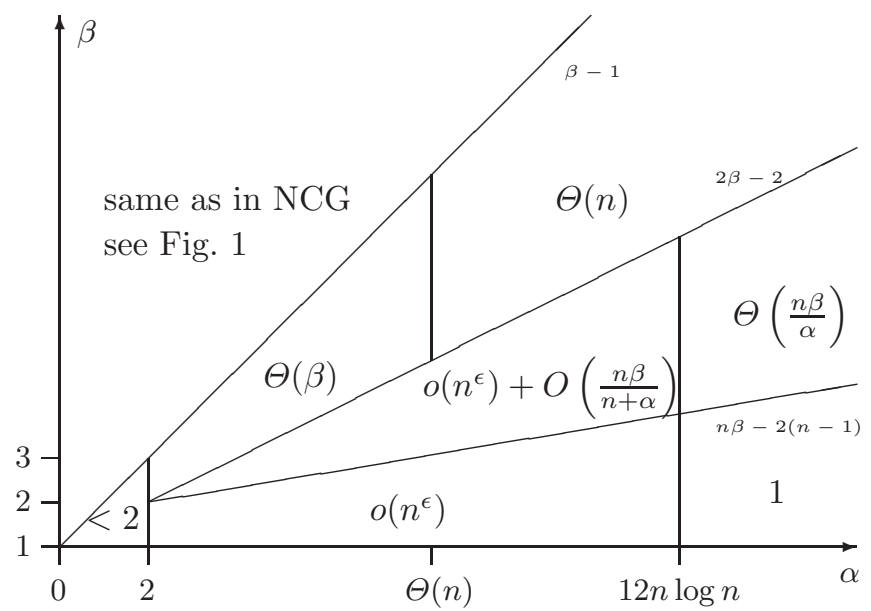

Fig. 3. Price of anarchy in the PCG

\section{$5 \quad$ Strong Equilibria}

In this section we assume agents are able to jointly deviate to different strategies. As stability concept we consider the strong equilibrium [4], in which no coalition $C$ of players can decrease the cost for each of its members by taking a joint deviation. More formally, if a state $s$ is a strong equilibrium (SE), then for each coalition of players $C$ and each possible strategy profile $s_{C}^{\prime}$ for the players in $C$ it holds that if there is a player $i \in C$ with $c_{i}\left(s_{C}^{\prime}, s_{-C}\right)<c_{i}(s)$, then there is another player $j \in C$ with $c_{j}\left(s_{C}^{\prime}, s_{-C}\right) \geq c_{i}(s)$. The price of anarchy for SE is a direct adaption of the price for NE and was studied in [3] for the NCG. The next theorem summarizes structural and existence properties of SE in the PCG. It shows, in particular, that with the exception of a small range of parameter values strong equilibria always exist in the PCG. Finally, the main result in this section is a general constant upper bound on the price of anarchy for SE in the PCG.

Theorem 5. For $\alpha<\beta-1$ the SE of the PCG are exactly the SE of the corresponding NCG. For $\alpha \geq \beta-1$ the social optimum in the PCG is a SE for all parameter values except $\beta<3$, and $\beta n-2 n+2-(\beta-1)<\alpha<\beta n-2 n+2$.

Theorem 6. The price of anarchy for SE in the PCG is at most 4.

\section{References}

1. Ackermann, H., Briest, P., Fanghänel, A., Vöcking, B.: Who should pay for forwarding packets? In: Deng, X., Graham, F.C. (eds.) WINE 2007. LNCS, vol. 4858, pp. 208-219. Springer, Heidelberg (2007)

2. Albers, S., Eilts, S., Even-Dar, E., Mansour, Y., Roditty, L.: On Nash equilibria for a network creation game. In: Proc. 17th SODA, pp. 89-98 (2006) 
3. Andelman, N., Feldman, M., Mansour, Y.: Strong price of anarchy. In: Proc. 18th SODA, pp. 189-198 (2007)

4. Aumann, R.: Acceptable points in general cooperative n-person games. In: Contributions to the Theory of Games IV. Annals of Mathematics Study, vol. 40, pp. 287-324 (1959)

5. Bala, V., Goyal, S.: A non-cooperative model of network formation. Econometrica $68,1181-1229(2000)$

6. Brandes, U., Erlebach, T. (eds.): Network Analysis: Methodological Foundations. LNCS Tutorial. Springer, Heidelberg (2005)

7. Corbo, J., Parkes, D.: The price of selfish behavior in bilateral network formation. In: Proc. 24th PODC, pp. 99-107 (2005)

8. Demaine, E., Hajiaghayi, M.T., Mahini, H., Zadimoghaddam, M.: The price of anarchy in network creation games. In: Proc. 26th PODC, pp. 292-298 (2007)

9. Dutta, B., Jackson, M.: The stability and efficiency of directed communication networks. Review of Economic Design 5(3), 251-272 (2000)

10. Eidenbenz, S., Kumar, A., Zust, S.: Equilibria in topology control games for ad hoc networks. In: Proc. DIALM-POMC Workshop Foundations of Mobile Comp., pp. $2-11(2003)$

11. Fabrikant, A., Luthera, A., Maneva, E., Papadimitriou, C., Shenker, S.: On a network creation game. In: Proc. 22nd PODC, pp. 347-351 (2003)

12. Freeman, L.: Centrality in social networks: Conceptual clarification. Social Networks 1(3), 215-239 (1979)

13. Jackson, M.: A survey of models of network formation: Stability and efficiency. In: Demange, G., Wooders, M. (eds.) Group Formation in Economics; Networks, Clubs and Coalitions, ch. 1, pp. 11-57. Cambridge University Press, Cambridge (2004)

14. Jackson, M., Wolinsky, A.: A strategic model of social and economic networks. Journal of Economic Theory 71(1), 44-74 (1996)

15. Koutsoupias, E., Papadimitriou, C.: Worst-case equilibria. In: Proc. 16th STACS, pp. 404-413 (1999)

16. Monderer, D., Shapley, L.: Potential games. Games and Economic Behavior 14, 1124-1143 (1996)

17. Moscibroda, T., Schmid, S., Wattenhofer, R.: On the topologies formed by selfish peers. In: Proc. 25th PODC, pp. 133-142 (2006)

18. Rivlin, E., Botafogo, R., Shneiderman, B.: Navigating in hyperspace: designing a structure-based toolbox. Comm. ACM 37(2), 87-96 (1994)

19. Valente, T., Foreman, R.: Integration and radiality: measuring the extent of an individual's connectedness and reachability in a network. Social Networks 20(1), 89-105 (1998) 